\title{
Margaret SCOTT, Fashion in the Middle Age
}

\section{Nadège Gauffre Fayolle}

\section{(2) OpenEdition \\ Journals}

Édition électronique

URL : https://journals.openedition.org/ccm/8152

DOI : $10.4000 / \mathrm{ccm} .8152$

ISSN : 2119-1026

\section{Éditeur}

Centre d'études supérieures de civilisation médiévale/Université de Poitiers

\section{Édition imprimée}

Date de publication : 1 septembre 2021

Pagination : 286-289

ISBN : 978-2-490783-10-6

ISSN : 0007-9731

\section{Référence électronique}

Nadège Gauffre Fayolle, "Margaret scotr, Fashion in the Middle Age », Cahiers de civilisation médiévale [En ligne], 255 | 2021, mis en ligne le 01 septembre 2021, consulté le 06 février 2022. URL : http:// journals.openedition.org/ccm/8152 ; DOI : https://doi.org/10.4000/ccm.8152

\section{(c) (i) $\odot$}

La revue Cahiers de civilisation médiévale est mise à disposition selon les termes de la Licence Creative Commons Attribution - Pas d'Utilisation Commerciale - Pas de Modification 4.0 International. 
Margaret Sсотт, Fashion in the Middle Age, Los Angeles, J. Paul Getty Museum Publications, 2018.

De mai à août 2011, au J. Paul Getty Museum de Los Angeles, s'est tenue une exposition consacrée au vêtement médiéval, intitulée Fashion in the Middle Ages. L'ouvrage éponyme qui accompagnait cet événement, publié pour la première fois en 2011, a été réédité en 2018. L'historienne de l'art Margaret Scott, s'est intéressée depuis les années 1980 à la mode médiévale à travers les sources iconographiques dans un premier livre
(Margaret Scoтt, The History of Dress Series: Late Gothic Europe, 1400-1500, Londres, Mills and Boon, 1980). À partir des manuscrits conservés à la bibliothèque du J. Paul Getty Museum, M. Scott brosse une synthèse de l'histoire du costume du XII ${ }^{e} \mathrm{~s}$. jusqu'au tout début du XVI ${ }^{\mathrm{e}} \mathrm{S}$., en proposant en regard de certaines œuvres enluminées des pièces archéologiques provenant de l'Art Institute of Chicago, du monastère de Santa Maria la Real de las Huelgas à 
Burgos en Espagne ou encore du Kunsthistorisches Museum de Vienne en Autriche. Fashion in the Middle Ages n'est pas à proprement parler un catalogue d'exposition : il n'y a pas de notices et ce n'est pas une édition scientifique. Il s'agit d'une publication destinée au grand public qui privilégie l'image en reproduisant une centaine d'enluminures sur les 120 pages qui constituent le livre.

Le parti pris de l'exposition et de l'ouvrage est assez original. M. Scott ne propose pas seulement une histoire du vêtement car tout au long du livre, elle attire l'attention du lecteur néophyte sur l'importance et la nécessité de la critique des sources afin de produire une science historique. En effet, afin d'étudier la culture matérielle, et particulièrement le vêtement, l'image est souvent convoquée; elle est toutefois biaisée de bien des manières et ne doit pas toujours être prise au sens littéral. Les commanditaires et les artistes produisent un discours sur le monde qui les entoure : le manuscrit procède donc à la fois de leur propre vision de la société, ainsi que des codifications artistiques et symboliques de leur époque.

Les textes explicatifs de l'ouvrage sont très concis. Le livre est en effet structuré en trois chapitres introduits uniquement par une citation tirée d'une œuvre littéraire médiévale. Les sous-parties suivent immédiatement; elles débutent par une synthèse d'une demi-page avant de passer au commentaire des enluminures constituant des focus qui enrichissent le propos introductif. Chaque chapitre est clos par la présentation d'un manuscrit, et de la manière dont il traite le fait vestimentaire au fil des enluminures. M. Scott aborde trois thématiques dans ce livre : "S'habiller au quotidien », la plus longue, «S'habiller pour une fonction », et « S' habiller pour un ailleurs ou un autre temps ».

Le premier chapitre, « S'habiller au quotidien », est le plus ancré dans la vie de tous les jours des femmes et des hommes du Moyen Âge, puisqu'il traite de la confection, de la mode et de la morale. Les matières premières du vêtement sont tout d'abord passées en revue : les fibres végétales pour les sous-vêtements, puis les lainages et les soieries, en passant par les doublures en fourrure. Les représentations iconographiques des différents types d'étoffes restent toutefois difficiles à interpréter : M. Scott utilise les travaux de Lisa Monnas sur les soieries dans la peinture afin d'aider le lecteur à identifier le velours, cette étoffe qui va connaître un grand succès dans les garde-robes de l'aristocratie et faire la fortune de certaines villes italiennes aux XIV et $\mathrm{XV}^{\mathrm{e}} \mathrm{s}$. Les velours unis sont reconnaissables par les jeux d'ombres et de lumières qui courent sur le vêtement, telle la houppelande bleue du cavalier figuré dans le Prayer Book of Cardinal Albrecht of Brandenburg (JPGM, ms. Ludwig IX 19, fol. 302v.). Les velours façonnés, plus précieux, comportent des motifs propres à ces types de tissages, permettant de les identifier et de les rapprocher de certaines pièces archéologiques. Une chasuble de velours florentin de l'Art Institut of Chicago, tissé v. 1440 (inv. 1980.615) est ainsi mise en regard d'une enluminure du livre de prières du duc de Bourgogne Charles le Bon qui le représente revêtu d'une houppelande de velours au décor presque identique (JPGM, ms. 37, fol. 1v.). Les fourrures de prix comme le menu vair ou l'hermine font l'objet d'une représentation stylisée et codifiée tout au long du Moyen Âge, respectivement des écussons blancs et gris alternés et un fond blanc à taches noires. Les artistes ne se préoccupent pas dans The Morgan Picture Bible (JPGM, ms. Ludwig I 6) de reproduire la réalité, mais de donner une information sur l'appartenance à l'aristocratie de l'individu. Les matières premières, des plus humbles aux plus coûteuses, contribuent en effet à la définition de la hiérarchie sociale.

Les formes des vêtements et la mode sont ensuite abordées : entre le $\mathrm{XII}^{\mathrm{e}}$ et le $\mathrm{XV}^{\mathrm{e}} \mathrm{s}$., les vêtements initialement construits sur un modèle en $\mathrm{T}$ très large, tels ceux figurés dans The Morgan Picture Bible, évoluent vers des coupes complexes qui épousent parfaitement les corps. Le pourpoint tiré de The Historical Bible est un bon exemple : ce costume masculin qui apparait au début du XIV ${ }^{\mathrm{e}} \mathrm{s}$. est moulant, court, parfois rembourré au niveau du buste; on le porte avec des chausses ajustées (JPGM, ms. 1, vol. 2, fol. 138). Les innovations vestimentaires sont également le fruit des sensibilités esthétiques d'une époque et d'une région. Les surcots masculins sans manches, par exemple, marquent une mode nationale : au XIII ${ }^{\mathrm{e}} \mathrm{s}$, on ne les rencontre que dans les manuscrits produits en Espagne comme le Feudal Customs of Aragon (JPGM, ms. Ludwig XIV 6). On connaît quelques exemples archéologiques retrouvés lors de l'ouverture des sépultures royales au monastère Santa Maria de Huelgas de Burgos (Pellote d'Enrique I de Castille, inv. 00650540). Ce modèle va se diffuser dans toute l'Europe, avec des adaptations, au siècle suivant. A contrario, certaines créations vestimentaires restent 
cantonnées à une aire géographique et M. Scott d'évoquer quelques exemples de particularités régionales dans une Europe où la vêture est somme toute assez semblable d'un royaume à l'autre : les voiles de tête plissés des régions germaniques, le plissage marqué à la taille des robes et houppelandes des femmes d'Europe centrale, illustrés respectivement par les manuscrits World Chronicle (JPGM, ms. 33) et Barlaam and Josaphat (JPGM, ms. Ludwig XV 9).

Enfin la question morale et sociale du vêtement est envisagée. Dès l'origine, le vêtement, symbole de la chute d'Adam et Eve, est suspect : il encourage la luxure, les dépenses superflues et attise les vanités, comme l'illustre cette femme à la coiffure élaborée minaudant devant un miroir dans le Bute Psalter (JPGM, ms. 46 fol. 32v.). Il participe toutefois, quand son port est bien régulé, à l'ordonnancement de la société. Chacun se vêt selon son rang et son genre et le vêtement devrait, dans une société idéale, révéler l'âme de son propriétaire. La littérature s'empare volontiers de ces codes pour figurer des allégories. Un manuscrit du Romance of the Rose (JPGM, ms. Ludwig XV 7) montre en effet Avarice dans une tenue unie très simple, usée et rapiécée alors qu'Amour est revêtu d'une houppelande de soie figurée à très larges manches. Le vêtement est porteur de sens et permet de connaître et reconnaître les personnages comme le développe M. Scott au chapitre suivant.

Dans la partie « S'habiller pour une fonction », l'a. met l'accent sur les vêtements à forte connotation symbolique qui permettent de reconnaître facilement le rôle des personnes mises en scène. Ces codes reflètent évidemment une réalité, mais les enlumineurs peuvent les utiliser hors de leur contexte afin de souligner l'importance d'une fonction dans la narration. Les vêtements de dignité que portent les hommes et les femmes de pouvoir sont très présents dans les manuscrits commandés par l'aristocratie. Dans les Hours of Louis XII (JPGM, ms. 79a), Saint Louis est représenté coiffé d'une couronne, vêtu du mantel traditionnel du sacre taillé dans une étoffe bleue ornée de fleurs de lys et doublée d'hermine. Lorsqu'un roi doit apparaitre sur une enluminure, il n'est pas rare de le voir paré d'un vêtement cérémoniel complètement incongru pour l'activité qu'il pratique. Le roi d'un psautier (JPGM, ms. 14, fol. 5) participe à une partie de chasse revêtu d'un manteau de dignité, le chef ceint d'une couronne, et monté sur un palefroi caparaçonné comme pour un tournoi. Le vêtement de dignité féminin représenté v. 1400 dans un livre d'heures (JPGM, ms. Ludwig IX 4, fol. 105) est le surcot ouvert, doublé de fourrure dont le corps est agrémenté d'armes familiales ou de devises personnelles. Le vestiaire ecclésiastique est lui aussi très codifié. La dalmatique que portent les diacres pendant la messe, illustrée par une pièce archéologique retrouvée dans la tombe de Rodrigo Ximénez de Rada, est souvent associée dans les enluminures aux saints : c'est le cas du martyr illustrant l'initiale d'un antiphonaire de 1350-1375 (JPGM, ms. 53). Les vêtements du clergé régulier sont encore bien plus spécifiques : chaque ordre, en fonction des activités de ses moines et des valeurs qu'il promeut, possède le sien. Le bénédictin enluminé dans un bréviaire (JPGM, ms. 24, fol. 4) porte des habits noirs, témoignages de repentance et d'humilité. Les dominicains qui assistent à la mort de saint patron s'habillent d'un manteau noir ou brun et d'un scapulaire blanc sur une tunique blanche (JPGM, ms. 62). Quant aux franciscains, très attachés à la pauvreté, ils utilisent des laines non teintes et vont en sandales comme dans la représentation de Saint-François de Sienne (JPGM, ms. Ludwig IX 13, fol. 195v.). Les membres de ces deux derniers ordres ont souvent exercé en tant que professeurs dans les universités, mais n'ont pas pour autant adopté le vêtement académique, qui apparaît à partir du XIII ${ }^{\mathrm{e}} \mathrm{s}$. L'habit des enseignants comme des étudiants doit être digne et long comme celui des médecins Saint Côme et Saint Damien (JPGM, ms. Ludwig IX 6, fol. 218). La tenue de certains travailleurs est également illustrée. Les livrées distribuées aux serviteurs des maisons princières sont toujours à la mode et ne diffèrent en rien d'un vêtement du quotidien si ce n'est par un accessoire tel qu'un tablier ou un torchon comme le montre le Book of Hours of the Bedford Master (JPGM, ms. Ludwig IX 6, fol. 1). Le calendrier des Spinola Hours (JPGM, ms. Ludwig IX 18) qui ferme le chapitre permet d'étudier certaines tenues ou accessoires liés au monde agricole tout en insistant sur l'adaptation du vêtement à la saison. Les vêtements chargés de symbolique énumérés dans cette partie ont été figés dans le temps et utilisés inchangés pendant de nombreuses décennies, voire plusieurs siècles.

Dans le chapitre final, «S'habiller pour un ailleurs ou un autre temps », M. Scott s'interroge sur la manière dont les contemporains s'approprient, imaginent et retranscrivent dans les manuscrits 
les vêtements historiques et les costumes des pays lointains. Elle s'intéresse tout d'abord à la représentation des temps bibliques. Les artistes du Moyen Âge respectent et partagent des archétypes vestimentaires inspirés des manuscrits romans tardifs et des costumes moyen-orientaux. Si la vierge et les mages peuvent arborer des vêtements à la mode, le Christ et les saints seront revêtus de robes simples et longues, parfois ornées de motifs orientaux et occidentaux, et seront pieds nus. Afin de signifier les âges de la vie, on peut trouver les mages représentés dans des tenues à caractères historiques (JPGM, ms. 83) : le plus vieux en tenue biblique, celui entre deux âges à la mode du XIV ${ }^{\mathrm{e}} \mathrm{s}$. alors que le manuscrit est peint en 1470 , et le benjamin en costume contemporain. À la Renaissance, c'est par le vêtement antique que les peintres inscrivent une scène au temps de la Bible. Les mondes grecs et romains sont d'ailleurs souvent mis en scène dans la littérature et les enlumineurs utilisent les vêtements byzantins en tant que vêtements historiques : Alexandre le Grand en majesté dans le livre des anciennes histoires est figuré avec les attributs des empereurs de Constantinople (BL, Add. ms. 15268, fol. 203). The Book of Deeds of Alexander the Great (JPGM, ms. Ludwig IX 18) illustre tout à fait le mélange de vêtements contemporains, historiques et même exotiques et étrangers, signifiés par un accessoire comme le turban.
Ces images pluridimensionnelles, pas uniquement narratives, permettent de donner de l'épaisseur au récit.

Sur l'ensemble de l'ouvrage, deux points nous ont gêné. M. Scott indique que la coupe des costumes $\mathrm{du} \mathrm{XII}^{\mathrm{e}}$ s. serait incommode et deviendrait plus confortable et pratique au $\mathrm{XIV}^{\mathrm{e}} \mathrm{s}$. Cette analyse ne nous semble pas très juste. Les plis que relève l'a. aux emmanchures de certains vêtements dans les enluminures d'un psautier (JPGM ms. 14 fol. 4v.) et de la Morgan Picture Bible ne sont pas des plis de contention, mais d'aisance, qui sur un patron très simple permettent un mouvement non entravé du bras. D'autre part, le discours très assertif concernant les foyers de création puis la diffusion des modes, notamment celles dites françaises, doit être bien plus nuancé car l'état actuel des connaissances ne permet de proposer que des hypothèses. Malgré ces quelques raccourcis, peut-être dus au format très concis de l'ouvrage, Fashion in the Middle Ages est un livre de vulgarisation réussi : il aborde de nombreuses thématiques et la qualité des reproductions permet au lecteur de suivre le propos de l'a. qui lui fournit toutes les clefs pour décrypter les sources iconographiques.

Nadège Gaufre FAYOLle 